\title{
SARS-CoV-2 antibody seroprevalence in a large neuroimmunological patient cohort
}

\author{
Katharina Eisenhut ${ }^{1,2} \cdot$ Stefan Buchka $a^{3,4} \cdot$ Peter Eichhorn ${ }^{5} \cdot$ Harald Meier $^{4,6} \cdot$ Fady Albashiti $^{4,6} \cdot$ Ulrich Mansmann ${ }^{3,4}$.

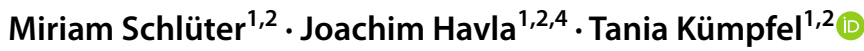

Received: 2 August 2021 / Revised: 22 September 2021 / Accepted: 22 September 2021 / Published online: 5 October 2021

(c) The Author(s) 2021

\section{Dear Sirs,}

The safety of patients with neuroimmunological conditions such as multiple sclerosis (MS) during the current SARS$\mathrm{CoV}-2$ pandemic has been a subject of major debate [1] and an accurate estimation of the burden of SARS-CoV-2 infection is pivotal. However, given the high prevalence of asymptomatic patients, the imperfect sensitivity of polymerase chain reaction (PCR) assays performed at a single time point, and limited testing capacity, the true number of SARS-CoV-2 infections likely exceeds the reported one. Serosurveys for SARS-CoV-2 antibodies (Abs) have so far only been reported for few neuroimmunological patient cohorts and often with only suboptimal assays $[2,3]$.

Here, we investigate the seroprevalence of SARS-CoV-2 Abs among neuroimmunology outpatient clinic patients from May 14th to September 30th, 2020, by two different assays and compare it to the seroprevalence of the general population. Further, precautionary health behavior was

Joachim Havla and Tania Kümpfel contributed equally.

Tania Kümpfel

tania.kuempfel@med.uni-muenchen.de

1 Institute of Clinical Neuroimmunology, University Hospital, LMU Munich, Marchioninistr. 15, 81377 Munich, Germany

2 Biomedical Center (BMC), Faculty of Medicine, LMU Munich, Martinsried, Germany

3 Institute of Medical Information Processing, Biometry, and Epidemiology, Faculty of Medicine, LMU Munich, Munich, Germany

4 Data Integration for Future Medicine (DIFUTURE) Consortium, LMU Munich, Munich, Germany

5 Institute of Laboratory Medicine, University Hospital, LMU Munich, Munich, Germany

6 Medical Technology and IT (MIT), University Hospital, LMU Munich, Munich, Germany assessed to understand how possible over- or under-cautious demeanor may affect seroprevalence.

Of all patients who were admitted to the neuroimmunology outpatient clinic of LMU Hospital in Munich, Germany, during mentioned period, 509 gave written informed consent to participate in the study. A serum sample for SARS-CoV-2 antibody serology was taken from all included patients. Clinical data were retrospectively obtained using structured documentation of routine clinical data. Precautionary health behavior data was acquired from a digital analyzable, paper-based questionnaire (Online Resource 1) which was answered by 474 out of 509 included patients. In the questionnaire, adherence to official recommendations concerning curfew, hygiene recommendations, and reduction of social contacts during and after the lockdown in Germany in spring 2020 (calendar weeks 12-19) was enquired by symmetrical, balanced Likert scales ranging from 1 ("not at all") to 5 ("very much"), respectively.

Each serum sample was tested for SARS-CoV-2 Ab by two assays at the Institute of Laboratory Medicine (LMU Hospital): Elecsys ${ }^{\circledR}$ Anti-SARS-CoV-2 electrochemiluminescence immunoassay system measuring pan-Immunoglobulin (ECLIA, Roche-Diagnostics, Basel, Switzerland; hereafter Ro-pan-Ig) and Anti-SARS-CoV-2 enzyme-linked immunosorbent assay measuring IgG only (ELISA, EUROIMMUN, Lübeck, Germany; hereafter Eu-IgG) [4]. While Ro-pan-Ig only detects antibodies against the SARS-CoV-2 nucleocapsid antigen, Eu-IgG exclusively binds Abs to the receptor binding domain (RBD). According to the manufacturer, Ro-pan-Ig has $99.5 \%$ sensitivity and $99.8 \%$ specificity $\geq 14$ days after a positive polymerase chain reaction (PCR) assay, respectively; the Eu-IgG sensitivity was $94.4 \%$ and specificity $99.6 \%$ [4]. 
Table 1 Cohort overview

\begin{tabular}{|c|c|}
\hline Characteristics & Patients $n=509$ \\
\hline \multicolumn{2}{|l|}{ Age [years] } \\
\hline$<18$ & $1(0.2 \%)$ \\
\hline $18-29$ & $66(13.0 \%)$ \\
\hline $30-39$ & $113(22.2 \%)$ \\
\hline $40-49$ & $127(25.0 \%)$ \\
\hline $50-59$ & $127(25.0 \%)$ \\
\hline $60-69$ & $54(10.6 \%)$ \\
\hline$\geq 70$ & $21(4.1 \%)$ \\
\hline \multicolumn{2}{|l|}{ Sex } \\
\hline $\mathrm{F}$ & $343(67.4 \%)$ \\
\hline M & $166(32.6 \%)$ \\
\hline \multicolumn{2}{|l|}{ Diagnosis } \\
\hline Autoimmune encephalitis & $16(3.1 \%)$ \\
\hline MS/CIS/RIS ${ }^{\mathrm{a}}$ & $345(67.7 \%)$ \\
\hline Myasthenia gravis & $23(4.5 \%)$ \\
\hline NMOSD & $34(6.7 \%)$ \\
\hline Other neuroimmunological disease & $91(17.9 \%)$ \\
\hline \multicolumn{2}{|l|}{ Immunotherapy } \\
\hline Immunomodulatory $^{\mathrm{b}}$ & $26(5.2 \%)$ \\
\hline Immunosuppressive $^{c}$ & $290(58.0 \%)$ \\
\hline B-cell-depleting therapy ${ }^{\mathrm{d}}$ & $152(30.4 \%)$ \\
\hline None & $184(36.8 \%)$ \\
\hline \multicolumn{2}{|l|}{ SARS-CoV-2 } \\
\hline History of (h/o) confirmed SARS-CoV-2 infection by PCR and/or serology $\mathrm{e}^{\mathrm{e}}$ & $3(0.6 \%)$ \\
\hline $\begin{array}{l}\text { H/o symptomatic COVID- } \\
19 \text { disease }^{\mathrm{f}}\end{array}$ & $2(0.4 \%)$ \\
\hline Positive SARS-CoV-2 Ab serology in our study ${ }^{\mathrm{g}}$ & $11(2.2 \%)$ \\
\hline Estimated SARS-CoV-2 Ab seroprevalence (Ro-pan-Ig and Eu-IgG) ${ }^{\mathrm{h}}$ & $1.27 \%$; CI $0.46-2.44$ \\
\hline Sensitivity- and specificity-adjusted SARS-CoV-2 Ab seroprevalence (Ro-pan-Ig) ${ }^{\mathrm{i}}$ & 1.58\%; CI 0.49-2.67 \\
\hline
\end{tabular}

${ }^{a}$ EDSS mean: 3.1 ; range: $0-8.0$

${ }^{\mathrm{b}}$ Beta-interferon, Glatiramer acetate

${ }^{c}$ Anakinra, Azathioprine, Canakinumab, Cladribine, Dimethyl fumarate, Eculizumab, Fingolimod, Infliximab, intravenous immunoglobulins (IVIG), Mycophenolic acid (MFA), Methotrexate (MTX), Natalizumab, Ocrelizumab, oral steroids, Rituximab, Teriflunomide, Tocilizumab (in alphabetical order)

${ }^{\mathrm{d}}$ Ocrelizumab, Rituximab. Only two patients on anti-CD20 therapy were seropositive for SARS-CoV-2 antibodies in our study and both infections were asymptomatic

${ }^{\mathrm{e}}$ All patients with a h/o SARS-CoV-2 infection were tested positive for SARS-CoV-2 Ab in our study

${ }^{\mathrm{f}}$ Of the two symptomatic patients, one was on immunotherapy with Anakinra, the other did not receive any immunotherapy

${ }^{g}$ Unadjusted seroprevalence considering patients positive for SARS-CoV-2 Ab in either Ro-pan-Ig or Eu$\mathrm{IgG}$ or both assays

${ }^{\mathrm{h}}$ See approach (ii)

${ }^{\mathrm{i}} \mathrm{See}$ approach (i)

Statistical analyses were performed using R-studio version 4.0.2 and WinBUGS (V 1.4.3, Imperial College and MRC, UK). To estimate the seroprevalence two different approaches were applied: (i) For comparison with the seroprevalence of the general population in Munich, which was reported earlier using the Ro-pan-Ig assay only [5], Ro-pan-Ig seroprevalence in our cohort was adjusted for the 


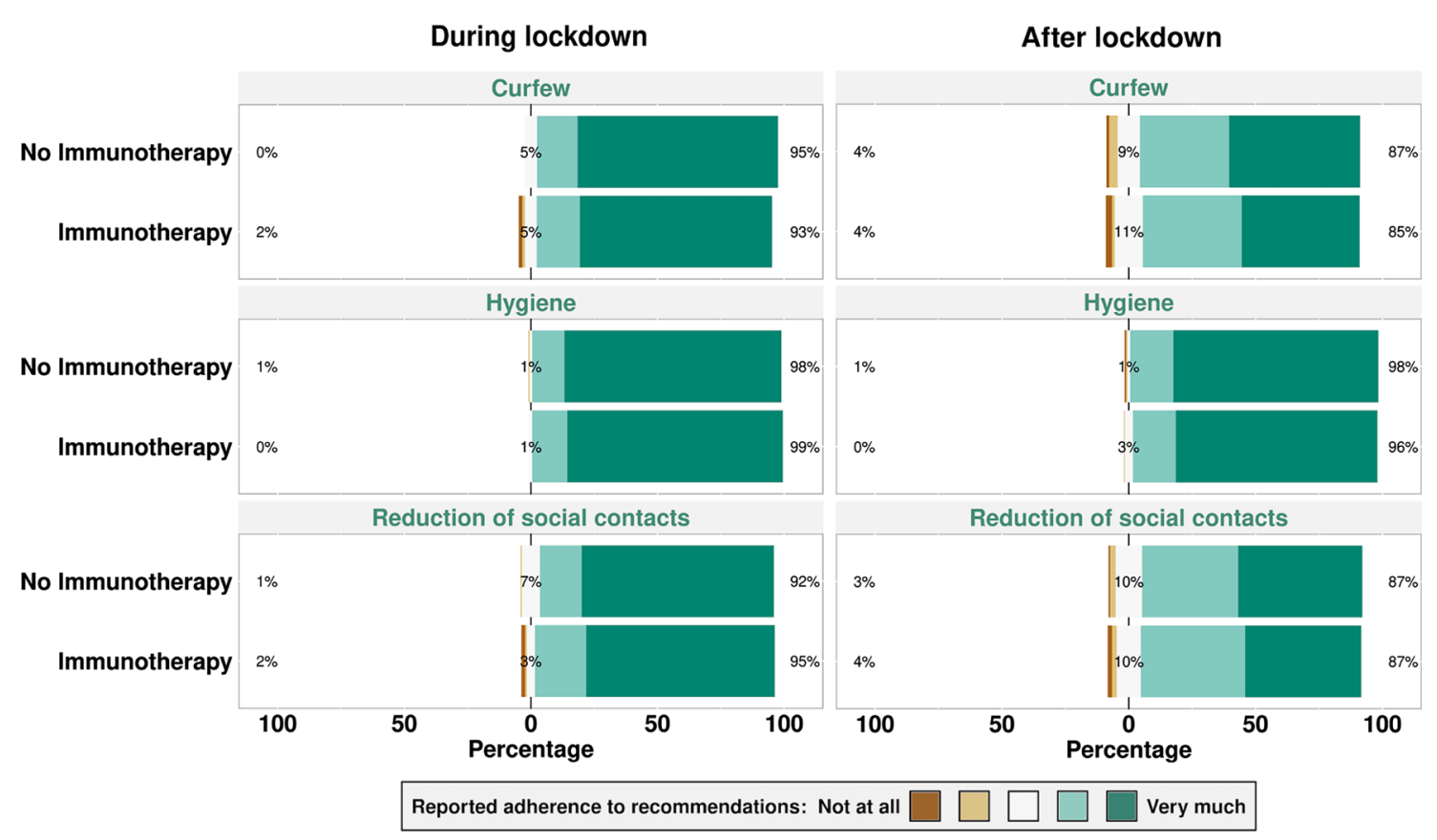

Fig. 1 Self-reported precautionary health behavior of neuroimmunology outpatient clinic patients with and without immunotherapy during and after the first lockdown in Germany

manufacturer's sensitivity and specificity. $95 \%$ confidence intervals (CI) were calculated by Poisson distribution.

(ii) Additionally, for a more robust estimation of SARS-

$\mathrm{CoV}-2-\mathrm{Ab}$ seroprevalence in our study population, a Bayesian approach was applied which considers both Ro-pan-Ig and Eu-IgG Ab assay results [6].

To compare two seroprevalences, a $\mathrm{Chi}^{2}$ test was applied. Questionnaire data were automatically retrieved by EvaSys software (ElectricPaper Evaluationssysteme, Lüneburg, Germany) and group comparisons were conducted by clustered Wilcoxon rank sum test.

Cohort details are provided in Table 1. In total, 11 out of 509 patients were identified as seropositive for SARSCoV-2 Ab by either Ro-pan-Ig $(n=4)$ or Eu-IgG $(n=2)$ or both assays $(n=5)$, yielding an estimated seroprevalence of $1.27 \%$ (CI 0.46-2.44; approach (ii)) in our cumulated patient cohort (cumulated seroprevalence; cSP). The sensitivity- and specificity-adjusted cSP regarding Ro-pan-Ig only test results was slightly higher (1.58\%; CI 0.49-2.67; approach (i)). The latter was compared to the SARS-CoV-2 Ab seroprevalence of the general population in Munich (1.82\%; CI 1.28-2.37; Ro-pan-Ig), as obtained from the Representative COVID-19 Cohort Munich [5]. Even though a tendency can be observed with a slightly lower cSP in our patient cohort, this difference was not significant $\left(\mathrm{Chi}^{2}\right.$ test, $\left.p=0.96\right)$.

Six of all 11 seropositive patients received immunotherapy at the time of serum sampling, among whom two were B-cell depleted due to anti-CD20 therapy. The sensitivity- and specificity-adjusted seroprevalence for Ro-panIg of all patients receiving immunotherapy at the time of serum sampling (iSP) was $1.39 \%$ (CI 0.09-2.69; approach (i)). Compared to the SARS-CoV-2 Ab seroprevalence of the general population in Munich [5], iSP was lower, but again not significantly reduced $\left(\mathrm{Chi}^{2}\right.$ test, $\left.p=0.77\right)$.

All seropositive patients were either asymptomatic $(81.8 \% ; n=9)$ or had a mild disease course $(18.2 \% ; n=2)$, according to the WHO clinical progression scale for COVID-19 research $($ score $<4)$ [7]. A prior SARS-CoV-2 infection was previously unknown in 8 out of 11 SARS$\mathrm{CoV}-2 \mathrm{Ab}$ positive patients, yielding $72.7 \%$ incidental cases among all seropositives in our study population.

Our cohort largely adhered to official regulations both during and after the first lockdown in Germany (Fig. 1) without significant differences between participants with and without immunotherapy (clustered Wilcoxon rank sum test, $p=0.63$ ). Of note, also all 11 SARS-CoV-2 Ab seropositive patients strictly adhered to recommendations as assessed in our study. 
Overall, the seroprevalence of SARS-CoV-2 Ab of neuroimmunological patients did not differ significantly from the seroprevalence of the general population. The high rate of incidental seropositives $(72.7 \%)$ including two patients on anti-CD20 treatment further highlights that a serology study may be useful for estimating the true burden of SARS-CoV-2 infection also in patients undergoing immunotherapy. Nevertheless, false-positive Ab results, particularly due to cross-reaction with other endemic coronaviruses, should be considered [8]. Inter-assay discrepancies may be due to different test reactivities, as reported previously [4].

In accordance with previous data [9], precautionary health behavior recommendations were strictly adhered to in our whole cohort which may have contributed to the low number of infections in our vulnerable patient population. Also, self-reported behavior was independent of immunotherapy use, suggesting a general tendency toward cautious health behaviors during the current pandemic in our patient cohort.

This study has limitations. First, the Representative Covid-19 Cohort Munich Study used for reference was conducted from April to June 2020 and, thus, does not correspond exactly with the time period of our study. Comparison of seroprevalence is further limited due to different cutoffs for the Ro-pan-Ig assay (0.4 in the Representative Covid19 Cohort Munich Study instead of 1.0 as intended by the manufacturer and as applied in this study). Also, seroprevalence in mentioned study may have been underestimated since they exclusively relied on Ro-pan-Ig assay results in the final analysis. Furthermore, in our study, precautionary health behavior was self-reported and appropriate controls are lacking, restricting any direct causal link between behavior and seroprevalence. Generally, the presented data are limited to a low-prevalence setting. Nonetheless, this study emphasizes the importance of serosurveys as a public health practice to avoid underestimation of SARS-CoV-2 infection burden in patients with neuroimmunological disease.

Supplementary Information The online version contains supplementary material available at https://doi.org/10.1007/s00415-021-10818-w.

Acknowledgements The authors would like to thank Sabine Lüngen, Eva Oswald and Angelika Bamberger for their active support of the project.

Author contributions KE: Formal analysis, Investigation, Resources, Data curation, Writing-Original draft, Writing-Review and Editing, Visualization; SB: Software, Formal analysis, Visualization; HM: Resources, Data curation; FA: Resources, Data curation; UM: Formal analysis, Writing-Review and Editing, Supervision; PE: Writing-Review and Editing, Supervision; MS: Conceptualization, Data acquisition, Review and Editing JH: Conceptualization, Methodology, Writing-Review and Editing, Supervision; TK: Conceptualization, Writing-Review and Editing, Supervision.

Funding Open Access funding enabled and organized by Projekt DEAL. This study was partially funded by the Friedrich-Baur-Stiftung (MS). JH, FA, SB and HM are (partially) funded by the German Federal Ministry of Education and Research (Grant Numbers 01ZZ1603[A-D] and 01ZZ1804[A-H] (DIFUTURE)).

Availability of data and material The datasets generated and analyzed during the study are available from the corresponding author on reasonable request.

Code availability Not applicable.

\section{Declarations}

Conflicts of interest MS, KE, SB, HM, FA, UM, and PE report no disclosures relevant to the manuscript. JH reports grants for OCT research from the Friedrich-Baur-Stiftung and Merck, personal fees and non-financial support from Celgene, Merck, Alexion, Novartis, Roche, Santhera, Biogen, Heidelberg Engineering, Sanofi Genzyme and nonfinancial support of the Guthy-Jackson Charitable Foundation, all outside the submitted work. TK has received travel expenses and personal compensation from Bayer Healthcare, Teva Pharma, Merck, Novartis Pharma, Sanofi-Aventis/Genzyme, Roche, and Biogen, as well as grant support from Bayer Schering AG, Novartis, and Chugai Pharma, all outside the submitted work.

Ethical approval The study was performed in accordance with the Helsinki II Declaration and approved by the ethics committee of the medical faculty of LMU Munich (project no 20-1081).

Consent to participate All participants gave written informed consent prior to inclusion into the study.

Consent for publication We thank all included participants for their consent to publish the study.

Open Access This article is licensed under a Creative Commons Attribution 4.0 International License, which permits use, sharing, adaptation, distribution and reproduction in any medium or format, as long as you give appropriate credit to the original author(s) and the source, provide a link to the Creative Commons licence, and indicate if changes were made. The images or other third party material in this article are included in the article's Creative Commons licence, unless indicated otherwise in a credit line to the material. If material is not included in the article's Creative Commons licence and your intended use is not permitted by statutory regulation or exceeds the permitted use, you will need to obtain permission directly from the copyright holder. To view a copy of this licence, visit http://creativecommons.org/licenses/by/4.0/.

\section{References}

1. Sharifian-Dorche M, Sahraian MA, Fadda G et al (2021) COVID19 and disease-modifying therapies in patients with demyelinating diseases of the central nervous system: a systematic review. Mult 
Scler Relat Disord 50:102800. https://doi.org/10.1016/j.msard. 2021.102800

2. Capasso N, Palladino R, Montella E et al (2020) Prevalence of SARS-CoV-2 antibodies in multiple sclerosis: the hidden part of the iceberg. J Clin Med 9(12):4066. https://doi.org/10.3390/jcm91 24066

3. van Kempen ZLE, Strijbis EMM, Al MMCT et al (2021) SARSCoV-2 antibodies in adult patients with multiple sclerosis in the Amsterdam MS Cohort. JAMA Neurol 78(7):880-882. https:// doi.org/10.1001/jamaneurol.2021.1364

4. Zilla M, Wheeler BJ, Keetch C et al (2021) Variable performance in 6 commercial SARS-CoV-2 antibody assays may affect convalescent plasma and seroprevalence screening. Am J Clin Pathol 155(3):343-353. https://doi.org/10.1093/ajcp/aqaa228

5. Pritsch M, Radon K, Bakuli A et al (2021) Prevalence and risk factors of infection in the representative COVID-19 cohort Munich. Int J Environ Res Public Health 18(7):3572. https://doi.org/10. 3390/ijerph18073572
6. Joseph L, Gyorkos TW, Coupal L (1995) Bayesian estimation of disease prevalence and the parameters of diagnostic tests in the absence of a gold standard. Am J Epidemiol 141(3):263-272. https://doi.org/10.1093/oxfordjournals.aje.a117428

7. WHO Working Group on the Clinical Characterisation and Management of COVID-19 infection (2020) A minimal common outcome measure set for COVID-19 clinical research. Lancet Infect Dis 20(8):e192-e197. https://doi.org/10.1016/S1473-3099(20) 30483-7

8. Okba NMA, Müller MA, Li W et al (2020) Severe acute respiratory syndrome Coronavirus 2-specific antibody responses in coronavirus disease patients. Emerg Infect Dis 26(7):1478-1488. https://doi.org/10.3201/eid2607.200841

9. Alnajashi H, Jabbad R (2020) Behavioral practices of patients with multiple sclerosis during Covid-19 pandemic. PLoS ONE 15(10):e0241103. https://doi.org/10.1371/journal.pone.0241103 